\title{
Low-energy collective electronic excitations at metal surfaces
}

\author{
Vyacheslav M. Silkin ${ }^{1,2,3}$ and E. V. Chulkov ${ }^{1,2}$ \\ ${ }^{1}$ Donostia International Physics Center, Donostia/San Sebastian, Spain, \\ ${ }^{2}$ Departamento de Fisica de Materiales, Facultad de Ciencias Químicas, Universidad del Pais \\ Vasco/Euskal Herriko Unibertsitatea, Donostia/San Sebastian, Spain \\ ${ }^{3}$ IKERBASQUE, Basque Foundation for Science, Bilbao, Spain \\ E-mail:waxslavas@sc.ehu.es
}

Address : Donostia International Physics Center, Paseo Manuel de Lardizabal 4, 20018

Donostia/San Sebastian, Basque Country, Spain

Telephone : $+34-943018284$

Collective electronic excitations at metal surfaces are well known to play a key role in numerous phenomena, ranging from physics and material science to biology and medicine. From the fifties it is known [1] that at the planar vacuum/metal boundary a mode, called surface plasmon, may emerge with frequency, $\omega_{\mathrm{sp}}=\omega_{\mathrm{p}} / \sqrt{ } 2$ where $\omega_{\mathrm{p}}$ is the bulk plasmon frequency. This relation between the bulk and surface plasmon frequencies is a manifestation of the fact that in a long-wavelength limit both quantities are entirely determined by the bulk dielectric function properties. In particular, the surface plasmon properties at small momentum transfers can be studied on the base of a surface response function $\varepsilon$ [2] related to the bulk dielectric function as $g=(\varepsilon-1) /(\varepsilon+1)$.

In real metallic systems the bulk dielectric function is often very different from the predictions of the free-electron-gas mode. In particular, recently it was demonstrated that indeed in many materials, like normal [3], charge-density-wave [4,5] and superconducting [6-10] metals and intercalated graphite [11], additionally to the conventional bulk plasmon, there exists a number of other bulk collective modes characterized by significantly lower frequencies. In this contribution we analyze which impact the presence of such low-energy modes in the bulk may produce on collective electronic excitations at the surface.

\section{References}

[1] R.H. Ritchie, Phys. Rev. 106, 874 (1957).

[2] B.N.J. Persson and E. Zaremba, Phys. Rev. B 31, 1863 (1985).

[3] V.M. Silkin, I.P. Chernov, Yu.M. Koroteev, and E.V. Chulkov, Phys. Rev. B 80, 245114 (2009).

[4] M. N. Faraggi, A. Arnau, and V.M. Silkin, Phys. Rev. B 86, 035115 (2012).

[5] P. Cudazzo, M. Gatti, and A. Rubio, Phys. Rev. B 86, 075121 (2012).

[6] W. Ku, W. E. Pickett, R. T. Scalettar, and A. G. Eguiluz, Phys. Rev. Lett. 88, 057001 (2002).

[7] Y. Q. Cai et al., Phys. Rev. Lett. 97, 176402 (2006).

[8] A. Balassis, E.V. Chulkov, P.M. Echenique, and V.M. Silkin, Phys. Rev. 78, 224502 (2008). 